Check for updates

Cite this: RSC Adv., 2017, 7, 44226

Received 15th July 2017

Accepted 7th September 2017

DOI: 10.1039/c7ra07799h

rsc.li/rsc-advances

\title{
Simultaneous degradation of tetracycline by a microbial fuel cell and its toxicity evaluation by zebrafish $\dagger$
}

\author{
Ji Wang, ${ }^{\text {ab }}$ Ming-Fang He, ${ }^{b}$ Dalu Zhang, ${ }^{c}$ Ziyu Ren, ${ }^{d}$ Tian-shun Song ${ }^{\star a b}$ \\ and Jingjing $\mathrm{Xie}$ (iD) *abe
}

Tetracycline (TC) is the second most commonly used antibiotic despite its high toxicity and persistence. In this study, a new approach for the anaerobic biodegradation of TC in a microbial fuel cell (MFC), with glucose-TC mixtures as the substrate, under gradient acclimation conditions was explored. Within 7 days, approximately $79.1 \%$ of TC was degraded by the MFC. This value was higher than that obtained through a traditional anaerobic method (14.9\%). The TC degradation rates in MFCs with a closed circuit were $31.6 \%$ higher than those in MFCs with an open circuit. Furthermore, zebrafish assessment showed that no toxicity was observed after MFC treatment. Microbial community analysis was performed on the anode of the MFCs under gradient acclimation conditions, and the results showed that TC was effectively degraded by the synergy of fermentative bacteria, acid-producing bacteria and electrogenic bacteria. This work confirmed that the anaerobic biodegradation of TC by MFCs is a cost-effective and environmentally friendly method.

\section{Introduction}

Currently, the extensive use of pharmaceuticals is an environmental issue. Antibiotics are the most widely used in disease prevention and treatment. ${ }^{1}$ In addition, antibiotics can also be used as feed additives to promote the growth rate of livestock and poultry animals.,3 As the largest producer and user of antibiotics in the world, China produces up to $210000 \mathrm{t}$ of antibiotics annually, half of which are consumed by animals. ${ }^{4}$ Antibiotics are weakly absorbed and incompletely metabolized in the body of humans and animals, therefore approximately 40-90 percent of antibiotics consumed by humans or animals is excreted via urine and feces in the parent form or as metabolites. ${ }^{5}$ Furthermore, large amounts of antibiotics or their metabolites are released into the aquatic environment by leakage and wastewater discharge. ${ }^{6,7}$ Long-term exposure to antibiotic-contaminated environment not only increases the

${ }^{a}$ State Key Laboratory of Materials-Oriented Chemical Engineering, Nanjing Tech University, Nanjing 211816, PR China.E-mail: xiej@njtech.edu.cn; tshsong@njtech. edu.cn

${ }^{b}$ College of Biotechnology and Pharmaceutical Engineering, Nanjing Tech University, 30 South Puzhu Road, Nanjing 211816, PR China. Tel: +86 2558139939

'International Cooperation Division, China National Center for Biotechnology Development, Beijing, PR China

${ }^{d}$ Nanjing Foreign Language School, Nanjing, PR China

'Jiangsu National Synergetic Innovation Center for Advanced Materials (SICAM), Nanjing 211816, PR China

$\dagger$ Electronic supplementary information (ESI) available. See DOI: 10.1039/c7ra07799h risk of harm to humans and animals, but also affects chlorophyll synthesis, enzyme secretion and root growth in plants. ${ }^{8}$ Thus, treating antibiotic-contaminated effluents before they are discharged into the aquatic environment is necessary.

Given its the low cost and high efficiency, tetracycline (TC) is the second most commonly used antibiotics in human activities and livestock breeding.9.10 Typically, domestic waste water contains relatively low TC $\left(1 \mu \mathrm{g} \mathrm{L}^{-1}\right),{ }^{11}$ whereas hospital waste water has TC concentration of more than $100 \mu \mathrm{g} \mathrm{L}{ }^{-1} .{ }^{12}$ Traditional methods, such as advanced oxidation process, ${ }^{13,14}$ active carbon adsorption ${ }^{15}$ and membrane separation ${ }^{16}$ are frequently used to remove TC from aqueous solutions. However, these techniques present some shortcomings such as high operation costs, low efficiency, and generation of toxic by-products. Biological treatment remains the most common and economical approach for the treatment of TC contaminants in wastewater. ${ }^{17,18}$ However, absence or deficiency of the terminal electron acceptors may result in decreased removal rate for TC.

Using microbial fuel cells (MFCs) offers an environmentally friendly and promising method for converting organic matter into electrical energy. ${ }^{19}$ MFCs adopt the electrode as a continuous long-term electron acceptor for anaerobic microorganism, which enhances the degradation of organic matter under anaerobic conditions. ${ }^{20,21}$ Therefore, MFC can degrade a wide range of organic substrates, ranging from easily degradable organics ${ }^{22,23}$ to bio-refractory organics. ${ }^{24,25}$ Recently, some articles focused on assessing the ability of MFC to improve TC removal. ${ }^{26,27}$ However, its degradation products might be more toxic than the parent compounds during the degradation of 
antibiotics. ${ }^{28}$ Therefore, toxicity after MFC treatment needs to be further investigated.

In this study, a dual-chamber MFC inoculated with anaerobic microorganism was constructed to investigate the removal of TC in MFCs and the microbial activity of MFC with TC as substrate was analyzed. The toxicity of the TC degradation products after MFC treatment was then evaluated by using zebrafish (Danio rerio). These results are expected to provide an environmentally friendly method for the biodegradation of TC by MFC.

\section{Materials and methods}

\section{MFC construction and operation}

The dual-chamber MFC reactor was made of plexiglas material (net volume of $250 \mathrm{~mL}$ each). The two chambers were separated by a proton exchange membrane (Nafion117, Dupont Co., USA). The anode and cathode were made of graphite felt $(50 \mathrm{~mm} \times$ $50 \mathrm{~mm} \times 5 \mathrm{~mm}$, length $\times$ width $\times$ thickness). The anolyte used glucose medium (pH 7.0) consisting of $0.31 \mathrm{~g} \mathrm{~L}^{-1} \mathrm{NH}_{4} \mathrm{Cl}$, $11.53 \mathrm{~g} \mathrm{~L} \mathrm{~L}^{-1} \mathrm{Na}_{2} \mathrm{HPO}_{4} \cdot 12 \mathrm{H}_{2} \mathrm{O}, 2.77 \mathrm{~g} \mathrm{~L}^{-1} \quad \mathrm{NaH}_{2} \mathrm{PO}_{4} \cdot 2 \mathrm{H}_{2} \mathrm{O}$, $0.13 \mathrm{~g} \mathrm{~L}^{-1} \mathrm{KCl}$ and $1 \mathrm{~g} \mathrm{~L}^{-1}$ glucose. The catholyte containing $13.2 \mathrm{~g} \mathrm{~L}^{-1} \mathrm{~K}_{3} \mathrm{Fe}(\mathrm{CN})_{6}, 2.77 \mathrm{~g} \mathrm{~L}^{-1} \mathrm{NaH}_{2} \mathrm{PO}_{4} \cdot 2 \mathrm{H}_{2} \mathrm{O}, 0.13 \mathrm{~g} \mathrm{~L}^{-1} \mathrm{KCl}$, $11.53 \mathrm{~g} \mathrm{~L}^{-1} \mathrm{Na}_{2} \mathrm{HPO}_{4} \cdot 12 \mathrm{H}_{2} \mathrm{O}$ were added to the cathode chamber of MFC. The MFCs were operated at a fixed external resistor of $1000 \Omega$ and maintained at $25{ }^{\circ} \mathrm{C}$. All the experiments were carried out in duplicate under each experimental condition.

During the startup stage, the anode chamber of the MFC was inoculated with $5 \mathrm{~mL}$ anaerobic activated sludge and $245 \mathrm{~mL}$ glucose culture medium. When the performance of the MFC stabilized, the substrate was replaced with glucose-TC mixtures containing glucose mixed with different concentrations of TC under a gradient acclimation as shown in Table 1. After the startup and gradient acclimation stage, the $50 \mathrm{mg} \mathrm{L}^{-1} \mathrm{TC}$ without glucose was used as substrate, and the performance of the MFC with closed circuit (CC) and MFC with open circuit (OC) was studied, respectively. In addition, $5 \mathrm{~mL}$ anaerobic activated sludge with $245 \mathrm{~mL}$ anolyte containing $50 \mathrm{mg} \mathrm{L}^{-1} \mathrm{TC}$ with non-gradient acclimation (NA) was also studied as control group.

The CC had a fixed external resistor of $1000 \Omega$, whereas the OC groups had none. Moreover, the CC and OC groups had domestication cycle of five weeks, but NA group lacked five cycles and directly used activated sludge to degrade tetracycline.

\section{Analyses}

The concentrations of TC were measured using Agilent 1200 infinity series high-performance liquid chromatography

Table 1 The concentration of the glucose and tetracycline mixtures in MFC during acclimation stage

\begin{tabular}{llllllll}
\hline Acclimation stage & A & B & C & D & E & F & G \\
Glucose $\left(\mathrm{mg} \mathrm{L}^{-1}\right)$ & 1000 & 1000 & 1000 & 1000 & 1000 & 500 & 0 \\
Tetracycline $\left(\mathrm{mg} \mathrm{L}^{-1}\right)$ & 10 & 20 & 30 & 40 & 50 & 50 & 50
\end{tabular}

Table 2 Mobile phase compositions for gradient elution

\begin{tabular}{lllll}
\hline Elution time/min & 0 & 2 & 2.1 & 6 \\
$\mathrm{~A} / \%$ & 8 & 18 & 20 & 8 \\
$\mathrm{~B} / \%$ & 0 & 0 & 5 & 0 \\
$\mathrm{C} / \%$ & 92 & 82 & 75 & 92
\end{tabular}

(HPLC), with an Agilent Eclipse Plus C18 column $(4.6 \times 100 \mathrm{~mm}$,

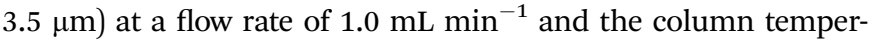
ature was $25^{\circ} \mathrm{C}$. The mobile phase consisted of $0.03 \%$ oxalic acid solution (A), methanol solution (B) and acetonitrile solution (C). The flow phase gradient used to obtain the measurements is shown in Table 2. The voltages were collected every $10 \mathrm{~min}$ with a precision multimeter with a data acquisition system (Keithley 2700 , USA). In order to get the polarization curves, the external resistance ranged from $5000 \Omega$ to $100 \Omega$. When the voltage output reached a steady value, current density and power density were calculated according to the projected anodic surface area. Current $(I)$ was calculated according to Ohm's law: $U=I R$, where $U$ is the voltage and $R$ is the external resistance. Power $(P)$ was calculated according to $P=I U$.

\section{Zebrafish maintenance and embryo collection}

Wild-type zebrafish (Tubingen line) were obtained from the Model Animal Research Center of Nanjing University. According to Truong et al. ${ }^{29}$ zebrafish should be kept at $28.5{ }^{\circ} \mathrm{C}$, with a 14:10 h light-dark photoperiod. The embryo medium was composed of $0.2 \mathrm{~g} \mathrm{~L}^{-1}$ of Instant Ocean ${ }^{\circledR}$ salt in distilled water with $0.01 \%$ methylene blue.

The authors declare that all procedures performed in studies involving live subjects were in accordance with the ethical standards of the institutional and national research committee and with the 1964 Helsinki declaration and its later amendments or comparable ethical standards. Authors also state that all the zebrafish studies performed were approved by the Institutional Animal Care and Use Committee (IAUC) of Nanjing Tech University.

\section{Embryo toxicity experiments}

The normal embryos were selected and placed in 48-well plates. Each well contained 10 embryos. The embryos were exposed to control, $50 \mathrm{mg} \mathrm{L}^{-1} \mathrm{TC}, \mathrm{CC}, \mathrm{OC}$ or NA group at $28.5^{\circ} \mathrm{C}$. The control group contained anodic liquid without TC. The $50 \mathrm{mg} \mathrm{L}^{-1} \mathrm{TC}$ group contained anodic liquid and $50 \mathrm{mg} \mathrm{L}^{-1}$ TC. For the accuracy of the experiment, each group was carried out in three replicates. At 72 hours post fertilization (hpf), the development of zebrafish embryos was monitored with a dissecting microscope (Nikon, SMZ745T). The measured data mainly included heart rate, hatching rate, survival rate, length of body and malformations. The heart rate was measured by counting the heart beating every 60 seconds under a dissecting microscope (Nikon, SMZ745T). The size of hydatoncus was measured with Photoshop CS5 (Adobe Systems, USA) for calculation of the pixels of the hydatoncus area. Dead zebrafish embryo was discarded every $12 \mathrm{~h}$. 


\section{Isolation and identification of bacterial strains}

For the isolation and purification of the TC-degrading microorganisms, an agar medium consisting of $0.3 \mathrm{~g} \mathrm{~L}^{-1}$ beef extract, $1 \mathrm{~g} \mathrm{~L}^{-1}$ peptone, $1.5 \mathrm{~g} \mathrm{~L}^{-1}$ of sodium chloride and $2 \%(\mathrm{w} / \mathrm{v})$ agar was prepared. The enriched sample was obtained from the biofilm on the anode surfaces in MFC under the $50 \mathrm{mg} \mathrm{L}^{-1} \mathrm{TC}$. The anode-associated biofilms were acquired by scraping the electrodes vigorously with a sterile razor blade. Then, $100 \mu \mathrm{L}$ of liquid samples were streaked on the fresh agar medium with $50 \mathrm{mg} \mathrm{L}{ }^{-1}$ TC. Single colonies were picked up and then transferred to the fresh agar medium. Bacterial genomic DNA was extracted, according to Jiang et al. reported. ${ }^{30}$ For bacterial $16 \mathrm{~S}$ rRNA gene, PCR reaction was carried out and the primers were $27 \mathrm{~F}$ and $1492 \mathrm{R}$. The products were sequenced by GENEWIZ (Suzhou, China). Sequencing results were compared with data in the NCBI database (http://ncbi.nlm.nih.gov/BLAST) by BLAST.

\section{DNA extraction for 16S rDNA illumina sequencing, and microbial community structure analysis}

The microbial community in the biofilm was analyzed by using bacterial 16S rDNA sequence analysis on an Illumina platform. Anodic biofilm samples were collected from MFC at the end of the experiment. Genomic DNA was extracted using the PowerSoil@ DNA Isolation Kit (MO BIO Laboratories Inc., Carlsbad, CA, USA), according to the manufacturer's protocol. DNA quality was assessed, at the ratios of 260/280 nm, using a Nano Drop ND-2000 Spectrophotometer (NanoDrop Technologies Inc., Wilmington, USA), and a highly pure genomic DNA (A260/A280 $\approx 1.8$ ) was used, only, for Illumina high-throughput sequencing by GENEWIZ (Suzhou, China). Richness and biodiversity indices were obtained via the Mothur software package. Similar sequences were clustered into operational taxonomic units (OTUs), on the basis of 3\% dissimilarity. Based on these clusters, rarefaction curves, OTUs, Chao1 richness estimations, Shannon diversity indexes and Good's coverages were generated, in MOTHUR version 1.30.0, for each sample (http://www.mothur.org/wiki/Main Page), at a cutoff of 0.03 , through the random selection of the minimum sequences of all our samples. The BLAST reports, of taxonomic classification, down to the phylum, class, order, family and genus levels were performed, using Mothur, based on the sequences from the Ribosomal Database Project (RDP), with a bootstrap cutoff of $50 \%$. An OTU table was generated and a heatmap, showing relative abundances, was created by HemI 1.0 (http://hemi.biocuckoo.org/index.php). ${ }^{31}$

\section{Results and discussion}

\section{Voltage production}

The experiment was divided into three stages. At stage I (startup phase), the anode in the MFC was colonized using a glucose solution (1000 $\left.\mathrm{mg} \mathrm{L}^{-1}\right)$. The MFC generated an initial circuit voltage of $150 \mathrm{mV}$ when $1000 \mathrm{mg} \mathrm{L}^{-1}$ glucose was immediately introduced (Fig. 1a). When the voltage decreased below $50 \mathrm{mV}$, the substrate was replaced and a second cycle was
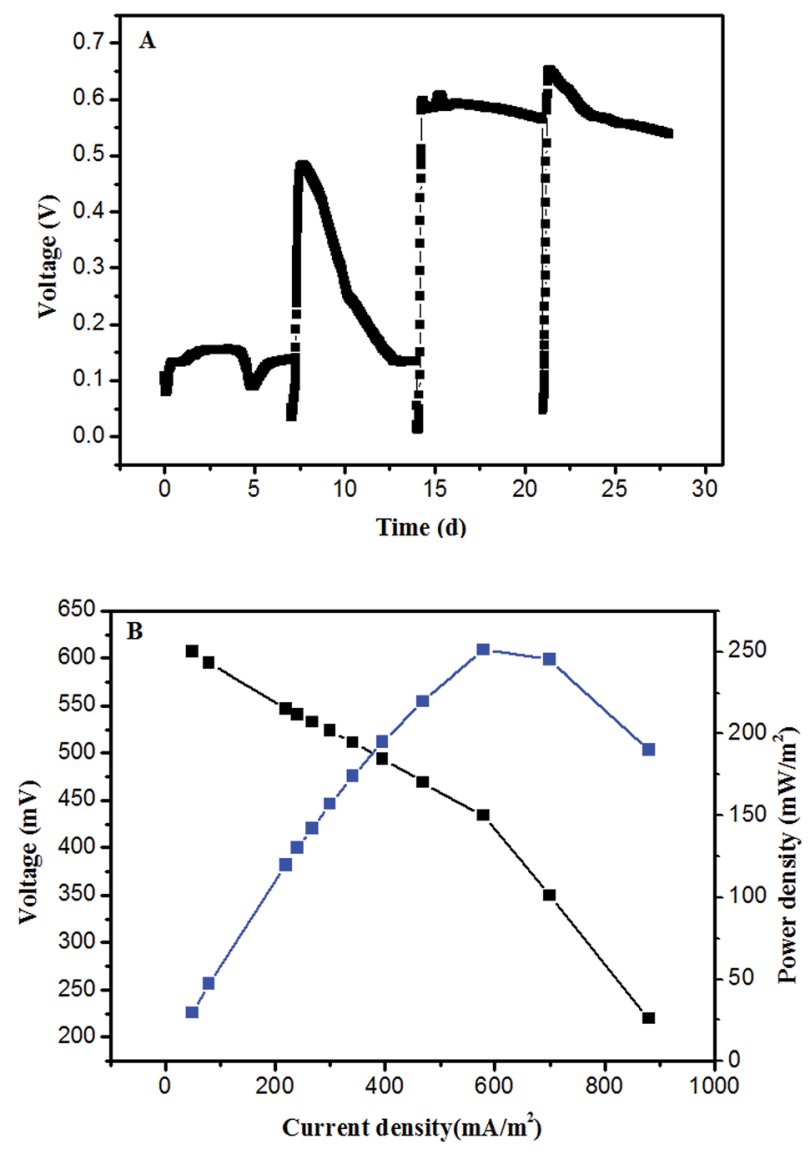

Fig. 1 (A) Voltage generation of MFC in start-up phase; (B) polarization curve and power density curves in the last cycle of start-up phase. Black line represents the voltage, and the blue line represents the power density.

initiated, the maximum voltage of $483 \mathrm{mV}$ was obtained. In the third cycle, the maximum voltage was $606 \mathrm{mV}$, and the voltage reached a relatively stable level $(600 \mathrm{mV})$ in the fourth cycle, the MFC generated the maximum power density of $251.14 \mathrm{~mW} \mathrm{~m}^{-2}$ at a current density of $578.7 \mathrm{~mA} \mathrm{~m}^{-2}$ (Fig. 1b). It indicated that the exoelectrogenic biofilm was formatted and the start-up of MFC had been completed.

At stage II (gradient acclimation), glucose-TC mixtures were introduced into the MFC with TC concentration of 10-50 $\mathrm{mg} \mathrm{L}^{-1}$ (Fig. 2). Voltages were obviously decreased when glucose-TC mixtures were added into the MFC at first. The initial voltage was $360 \mathrm{mV}$ and continued to decline. The result implied that TC might reduce the electrochemical activity of bacteria on the anode in MFC. After 7 day acclimation, the substrate was changed to $1 \mathrm{~g} \mathrm{~L}^{-1}$ glucose with $20 \mathrm{mg} \mathrm{L}^{-1} \mathrm{TC}$. The voltage of MFC started to rise slowly and the maximum voltage was $482 \mathrm{mV}$ on the 14th day. Further, the maximum voltage of MFC was $582 \mathrm{mV}$ at stage C and $536 \mathrm{mV}$ at stage D, indicating that the microorganisms on the anode slowly adapted to the TC environment. As the concentration of TC further increased to $50 \mathrm{mg} \mathrm{L}^{-1}$, the voltage of MFC became stable at stage $\mathrm{E}$, and the duration of electricity production was also prolonged. It demonstrated that microorganisms on the 


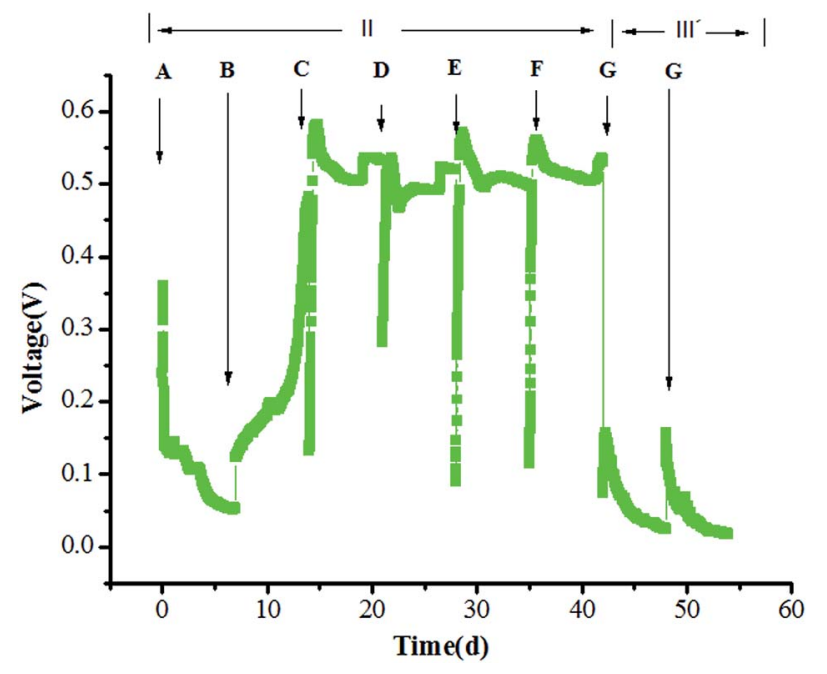

Fig. 2 Voltage output of MFC using glucose-TC mixtures containing glucose mixed with different concentrations of TC under an external resistance of $1000 \Omega$. (A) $1 \mathrm{~g} \mathrm{~L}^{-1}$ glucose $+10 \mathrm{mg} \mathrm{L}^{-1} \mathrm{TC}$; (B) $1 \mathrm{~g} \mathrm{~L}^{-1}$ glucose $+20 \mathrm{mg} \mathrm{L}^{-1} \mathrm{TC}$; (C) $1 \mathrm{~g} \mathrm{~L}^{-1}$ glucose $+30 \mathrm{mg} \mathrm{L}^{-1} \mathrm{TC}$; (D) $1 \mathrm{~g} \mathrm{~L}^{-1}$ glucose $+40 \mathrm{mg} \mathrm{L}^{-1} \mathrm{TC}$; (E) $1 \mathrm{~g} \mathrm{~L}^{-1}$ glucose $+50 \mathrm{mg} \mathrm{L}^{-1} \mathrm{TC}$. (F) $0.5 \mathrm{~g} \mathrm{~L}^{-1}$ glucose $+50 \mathrm{mg} \mathrm{L}^{-1} \mathrm{TC}$; (G) $50 \mathrm{mg} \mathrm{L}^{-1} \mathrm{TC}$; (stage II) glucose-TC mixtures were introduced into the MFC; (stage III) $50 \mathrm{mg} \mathrm{L}^{-1} \mathrm{TC}$ was used as the sole substrate for the MFC.

anode can be acclimated and is suitable for generating electricity in the presence of TC. At stage F, the concentration of TC remained $50 \mathrm{mg} \mathrm{L}^{-1}$ and the concentration of glucose decreased to $500 \mathrm{mg} \mathrm{L}^{-1}$, the maximum voltage was $561 \mathrm{mV}$, which was slightly less than the voltage at stage E. This also proved that the microorganisms on the anode has adapted in the presence of high TC concentrations, even in the case of reducing easily degradable organics. At stage III, $50 \mathrm{mg} \mathrm{L}^{-1} \mathrm{TC}$ was used as the sole substrate for the MFC. After running two cycles in the presence of $50 \mathrm{mg} \mathrm{L}^{-1} \mathrm{TC}$, the voltage of MFC rapidly decreased and the maximum voltage was respectively $157 \pm 2 \mathrm{mV}$, the generated voltage indicated that TC can be used as sole substrate to generate electricity by microorganisms on the anode.

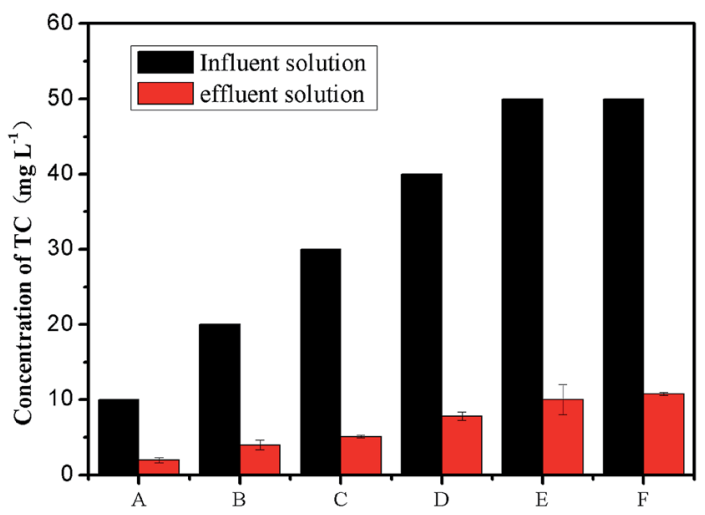

Fig. 3 TC concentration of influent solution and effluent solutions from stages $A$ to $F$.

\section{Degradation of TC}

The change in TC concentration at stage II is shown in Fig. 3. During the acclimation stage, the concentration of TC reduced from $10 \mathrm{mg} \mathrm{L}^{-1}$ to $1.9 \mathrm{mg} \mathrm{L}^{-1}$ at stage A. When the initial TC concentration was $20 \mathrm{mg} \mathrm{L}^{-1}$, the effluent concentration of TC was $4.0 \mathrm{mg} \mathrm{L}^{-1}$. As the TC concentration further increased to $40 \mathrm{mg} \mathrm{L}{ }^{-1}$, the removal rates of TC in both cycles was similar and nearly reached to $80 \%$. When the TC was $50 \mathrm{mg} \mathrm{L}^{-1}$ at stages $\mathrm{E}$ and $\mathrm{F}$, the effluent concentrations of TC was $9.9 \mathrm{mg} \mathrm{L}^{-1}$ and $10.7 \mathrm{mg} \mathrm{L}^{-1}$, respectively. This result showed that the removal of TC cannot be affected along with the glucose reduction, as the biofilm on anode adapted with the presence of the TC in gradient acclimation.

As was shown in Fig. 4a, the removal efficiency in the CC group was the highest. Approximately $53 \%$ of TC was removed in CC group within $12 \mathrm{~h}$. After 3 days, the removal efficiency of TC in CC group was more than $70 \%$. After 7 days running, TC concentration was only $10.5 \mathrm{mg} \mathrm{L}^{-1}$, and the removal efficiency of TC reached up to $79.1 \%$. For the OC group, $39.9 \%$ of TC
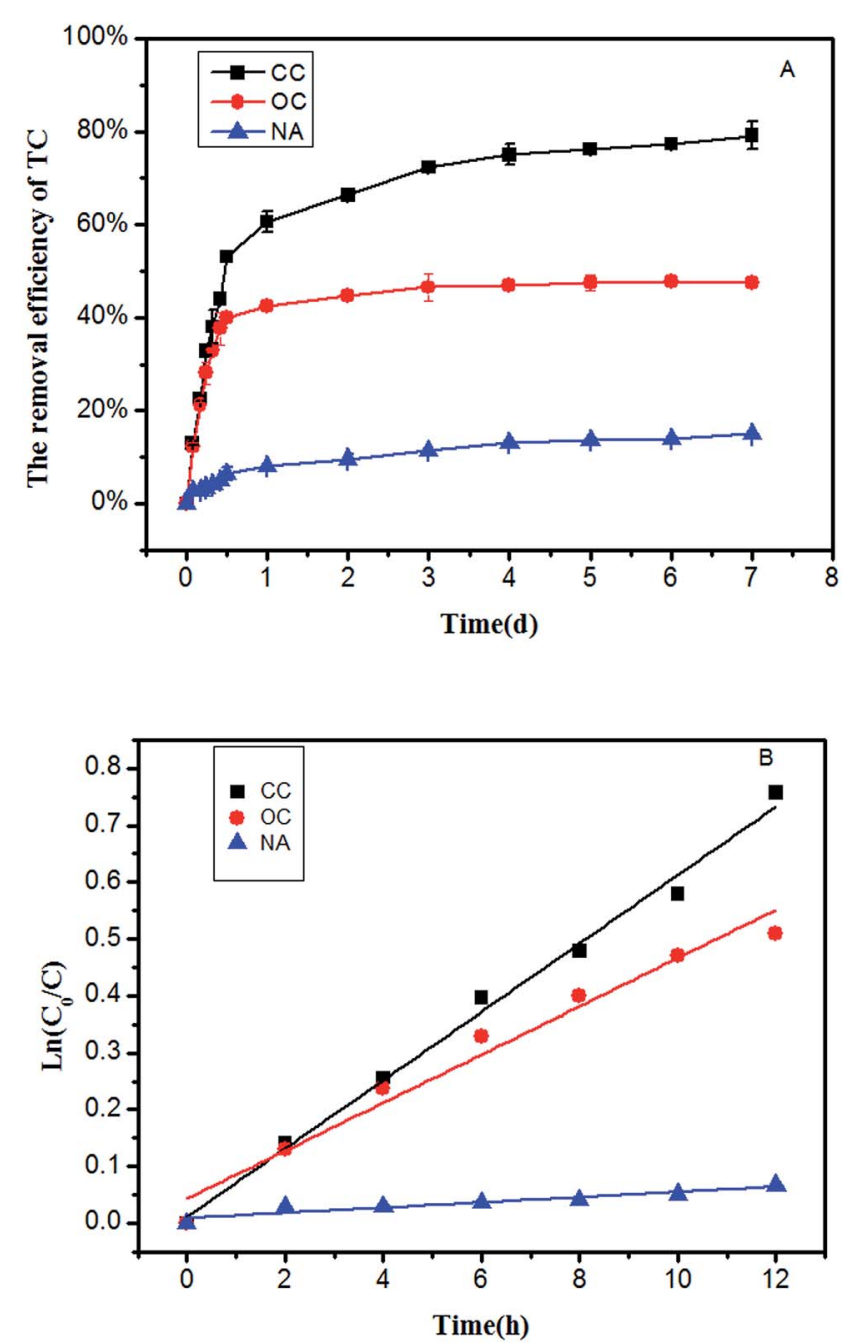

Fig. 4 (A) Removal efficiency of TC (B) kinetics curve of TC degradation in the CC, OC and NA groups when $50 \mathrm{mg} \mathrm{L}^{-1} \mathrm{TC}$ was fed as sole substrate. 
concentration was removed within $12 \mathrm{~h}$, and the removal efficiency of TC in the MFC was nearly constant in the subsequent days, and the final removal efficiency of TC was $47.5 \%$ within 7 days. On the contrary, no significant change was observed in the TC concentration of the NA group. Only $6.4 \%$ TC was removed within $12 \mathrm{~h}$, and the removal efficiency of TC in the NA reactors was $14.9 \%$ within 7 days. This phenomenon may also emphasize the importance of gradient acclimation in the process of TC removal by MFC.

To investigate the TC degradation capability among different groups, a degradation kinetics model was established within $12 \mathrm{~h}$. The substrate biodegradation was described with the following first-order kinetic model:

$$
\ln \left(C_{0} / C\right)=k \times t
$$

where $C$ is the concentration of $\mathrm{TC}, \mathrm{mg} \mathrm{\textrm {L } ^ { - 1 }} ; C_{0}$ is the initial concentration, $\mathrm{mg} \mathrm{L} \mathrm{L}^{-1} ; k$ is the biodegradation kinetic constant, $\mathrm{h}^{-1}$; and $t$ is the time, h. Fig. $4 \mathrm{~b}$ shows the results of the linear curve fit of degradation over time was demonstrated, which focused on the biodegradation kinetics of TC. $k_{\mathrm{CC}}$ was the highest $\left(0.060 \mathrm{~h}^{-1}, R^{2}=0.99\right)$, followed by $k_{\mathrm{OC}}\left(0.042 \mathrm{~h}^{-1}\right.$, $\left.R^{2}=0.97\right)$, and then $k_{\mathrm{NA}}\left(0.0046 \mathrm{~h}^{-1}, R^{2}=0.90\right)$. The $k$ value indicates that the degradation rate of TC in MFC with closed circuit is the fastest. The TC degradation rate in the CC group was 1.42 times than that of the OC group, and 13 times than that of the NA group.

Among all three groups, the CC group showed the highest TC removal rate, followed by OC and NA, which showed the lowest removal rate. It might be attributed to the enhanced phenol by MFC. Under the closed circuit, the current generated by the oxidation of TC on the anode was able to be transferred to the terminal electron acceptor oxygen through the external circuit, thereby completing the redox reaction and accelerating the degradation rate of TC. Although no current was present in the OC and NA groups, the final removal efficiency of TC for the OC group was $47.5 \%$, and that for NA group was $14.9 \%$. This huge difference might be mainly attributed to the differences between microbial activities with acclimation and those without. Notably, the removal rates can be distinguished as two different stages in all three groups. The first stage might be defined within $12 \mathrm{~h}$, while the remaining time during the entire process might be defined as stage two. In all three groups, the removal rate of TC at stage one was higher than that at stage two. At the first stage, the high removal rate might be attributed to the combined effects of both absorption and degradation on this TC removal process by MFC. However, at the second stage, the adsorption sites on the biofilm might have been gradually occupied, and the effect of electrode as a continuous long-term electronic acceptor might have been the main factor.

a


Fig. 5 (a) The photos of zebrafish embryos with exposure to different treatment group; (b) measurement of hydatoncus area value in different groups. 


\section{Zebrafish embryos toxicity assessment}

Zebrafish is a model vertebrate that has high homology with the human genome. Thus, it is an excellent model organism in various biomedical fields, especially for toxicity assessment, developmental biology ${ }^{32}$ and drug screening. ${ }^{33}$ Besides, compared with the traditional model animals such as mouse, zebrafish is lower in cost and has shorter cycle time and lower compound requirements. ${ }^{34}$ Although TC can be effectively degraded by MFC, the toxicity of the degradation products is unknown. The degradation products might be more toxic than the parent compounds during antibiotics degradation. Therefore, the toxicity of the effluent in each group was evaluated by zebrafish.

The hatching of the embryos seemed to display the different hydatoncus status in the different treatment groups among all the toxicity tests (Fig. 5). The size of hydatoncus in the embryo under $50 \mathrm{mg} \mathrm{L}^{-1}$ tetracycline was the highest, followed by those in the NA and OC group. The CC and control groups had the smaller size of hydatoncus. The area of hydatoncus in the control group was similar to that of the CC group, which were 2.1 and $2.2\left(\times 10^{4}\right.$ pixels $)$, respectively. However, compared with the control and CC groups, the NA, OC, and $50 \mathrm{mg} \mathrm{L}^{-1}$ groups had larger yolk sac edema areas at 3.0, 3.1, and $3.7\left(\times 10^{4}\right.$ pixels), respectively. The intuitionistic images results showed that the effluent did not cause any malformation after the MFC treatment. Further, some other important parameters of zebrafish were determined. Hatching rate is an important indicator for toxicity. As shown in Fig. 6a, the hatching rate of the CC group was the highest $(100 \%)$ similar to the control, followed by the OC (95.8\%) and NA (93.3\%), which was slightly higher than the $50 \mathrm{mg} \mathrm{\textrm {L } ^ { - 1 }}$ TC. The results showed that the effluent in the CC group had no effect on the hatching rate of zebrafish.

Survival rate is considered to be an important event within the course of embryogenesis and thus is a key endpoint for the embryo toxicity test. As shown in Fig. 6b, only $33.3 \%$ of the zebrafish survived in $50 \mathrm{mg} \mathrm{L}^{-1}$ TC. However, the survival rate of embryos treated by the CC, OC and NA groups were $96.7 \%$, $73.3 \%$ and $43.3 \%$, respectively. The effluent in the CC group showed no effect on the survival of zebrafish embryos in contrast to that of the control $(96.7 \%)$.

The heart rate of zebrafish embryos reveals cardiovascular toxicity. Fig. 6C showed that the heart rates of the zebrafish embryos were exposed to the different treatment groups at 72 hpf. The normal heartbeat of the control group was 151 times per minute. However, the heartbeats of the other groups were
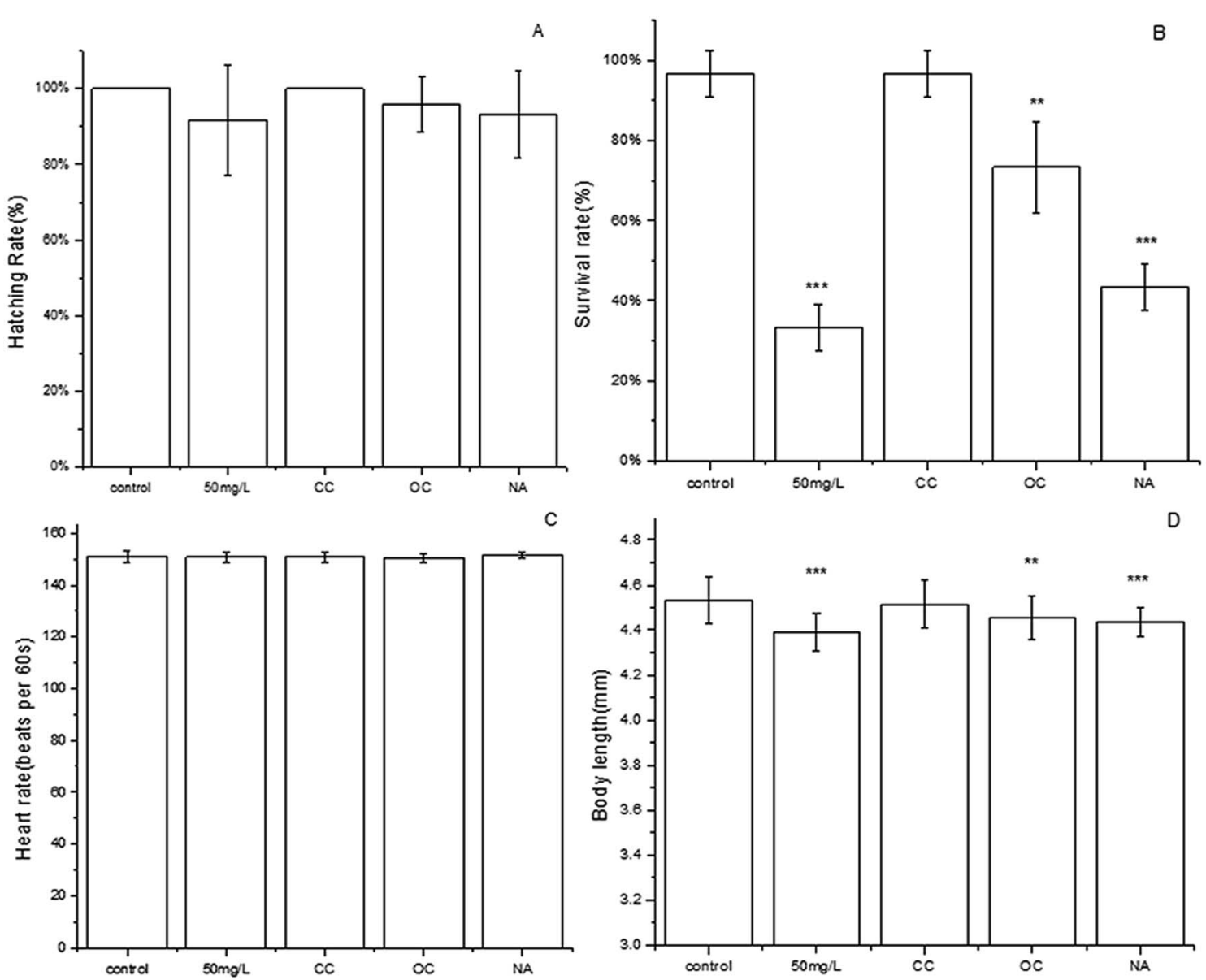

Fig. 6 Toxicity assessment of different treatment groups. (A) Hatching rate, (B) survival rate, (C) heart rate and (D) body length of zebrafish embryos. Each point represents a mean \pm standard error of the mean from a representative experiment. $* P<0.05, * * P<0.01$, and $* * * P<0.001$ in one-way ANOVA analysis followed by the Dunnett multiple comparison test. 
the same as those of the control in all cases. TC had no obvious effect on the heart rate at all test conditions.

Body length is a significant indicator of zebrafish embryo growth (Fig. 6d). In the control group, the body length of healthy zebrafish embryo in the control group was $4.53 \mathrm{~mm}$ at $72 \mathrm{hpf}$. The body length of zebrafish embryos exposed to CC, OC, NA and $50 \mathrm{mg} \mathrm{L}^{-1} \mathrm{TC}$ were $4.39 \mathrm{~mm}, 4.51 \mathrm{~mm}, 4.45 \mathrm{~mm}$ and 4.43 $\mathrm{mm}$. This phenomenon indicated that TC could shorten the body length of zebrafish, especially in the $50 \mathrm{mg} \mathrm{L}^{-1}$ group. However, compared with the OC and NA groups, the CC group had a better effect on the recovery of the body length of zebrafish.

The survival rate of the zebrafish seemed to display the most sensitivity to the TC with different treatment groups among the toxicity test parameters. The differences among the effects of these treatment groups attributed to some malformations caused by TC during embryogenesis. According to our zebrafish toxicity assessments, the CC group showed less toxicity than the other groups. The result showed that TC was effectively degraded by MFC with closed circuit and the resulting degradation products showed nontoxicity on zebrafish embryogenesis.

\section{Bacterial community composition}

As shown in Fig. 7a, the dominant phyla in the bacterial communities on anode was Proteobacteria, accounting for $62.4 \%$ of the total bacteria sequence, followed by the Firmicutes (26.1\%) in the microbial community. Bacteroidetes was also detected, in the biofilm on anode with an abundance of approximately $8.1 \%$. Additionally, the bacterial communities were also identified at genus level (Fig. 7b). The highest relativeabundance group in the microbial community of the inoculum was Achromobacter, with an abundance of $14.9 \%$, followed by Burkholderia-Paraburkholderia (12.3\%) and Alcaligenes (9.4\%), Geobacter (5.01\%), Stenotrophomonas (4.4\%) and Enterobacter (4\%). Achromobacter, which belongs to Proteobacteria, was reported to have the capability to degrade aromatic compounds, such as benzene, toluene, ethylbenzene and xylene. ${ }^{35,36}$ Burkholderiales, was reported to use organic carbon as an electron donor and to be capable of denitrification ${ }^{37}$ and production of nitrogen gas for the maintenance of anaerobic environment in an anode region. Alcaligenes, ${ }^{38}$ and Geobacter, ${ }^{39}$ which belongs to Proteobacteria, is an electrochemically active bacterium, responsible for electron transfer via an electrode. Stenotrophomonas is an acid-producing bacterium ${ }^{40}$ that can ferment carbohydrates to produce organic acid. Enterobacter has a certain drug-resistance, ${ }^{41}$ and further research was found that Enterobacter from drinking and recreational water sources exhibited varying antibiotic sensitivity. ${ }^{42}$ Correspondingly, one Enterobacter strain was isolated with TC as the sole carbon substrate from the anode at the end of our experiment (Fig. S1 $\dagger$ ). The results demonstrated that the Enterobacter in bacterial communities was most likely responsible for the biodegradation of TC in our MFC anodic region. In summary, the removal of TC might be the synergistic action of fermentative bacteria, acid-producing bacteria and electrogenic bacteria
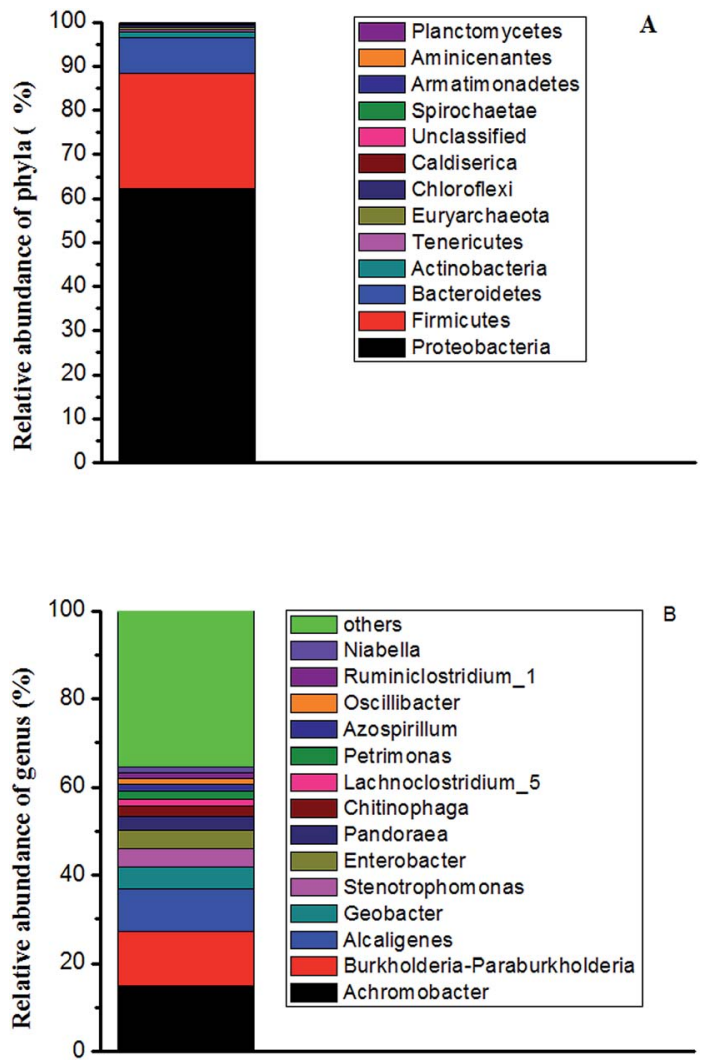

Fig. 7 At the end of the experiment, (A) relative abundance of bacterial phyla communities (B) genus communities in anode after MFC treatment TC.

on the anode. Given the limited understanding of the function of each strain in bacterial communities of TC removal in MFC system, future research should be focus on the understanding of the synergistic TC degradation mechanism of bacterial communities on the anode.

\section{Conclusion}

TC can be rapidly degraded in MFC reactors and the degradation rate of TC with different concentrations was basically stable at $80 \%$. The removal efficiency for TC by MFC with closed circuit was higher than that of MFC with opened circuit, and the traditional anaerobic method showed the lowest removal efficiency for TC. The results emphasized the importance of electron transport and the necessity of domestication for TC biodegradation. Zebrafish experiments illustrated that the effluent after MFC treatment was biocompatible and the degradation products showed no toxicity on zebrafish embryogenesis. This work confirmed that anaerobic biodegradation of TC by MFCs is a cost-effective and environmentally friendly method.

\section{Conflicts of interest}

There are no conflicts to declare. 


\section{Acknowledgements}

The National Science Fund of China (Grant No. 21390201); the Major projects of natural science research in Jiangsu Province (Grant No. 15KJA530002); Fund from the State Key Laboratory of Materials-Oriented Chemical Engineering (ZK201605, KL15-07), CAS Key Laboratory of Bio-based Materials (No. KLBM2016009) and the Priority Academic Program from Development of Jiangsu Higher Education Institutions.

\section{References}

1 M. Seifrtová, L. Nováková, C. Lino, A. Pena and P. Solich, Anal. Chim. Acta, 2009, 649, 158-179.

2 K. Kummerer, Chemosphere, 2009, 75, 417-434.

3 N. Le-Minh, S. J. Khan, J. E. Drewes and R. M. Stuetz, Water Res., 2010, 44, 4295-4323.

4 L. J. Zhou, G. G. Ying, S. Liu, J. L. Zhao and B. Yang, Sci. Total Environ., 2013, 452, 365-376.

5 K. Kumar, S. C. Gupta, Y. Chander and A. K. Singh, Adv. Agron., 2005, 87, 1-54.

6 N. H. Tran, J. Li, J. Hu and S. L. Ong, Environ. Sci. Pollut. Res., 2014, 21, 4727-4740.

7 Y. Luo, W. Guo, H. H. Ngo, L. D. Nghiem, F. I. Hai, J. Zhang, S. Liang and X. C. Wang, Sci. Total Environ., 2014, 473-474, 619-641.

8 M. Crane, C. Watts and T. Boudard, Sci. Total Environ., 2006, 367, 23-41.

9 S. Yahiat, F. Fourcade, S. Brosillon and A. Amrane, Int. Biodeterior. Biodegrad., 2011, 65, 997-1003.

10 P. Mahamallik, S. Saha and A. Pal, Chem. Eng. J., 2015, 276, 155-165.

11 S. Yang, J. Cha and K. Carlson, J. Chromatogr. A, 2005, 1097, 40-53.

12 A. Pena, M. Paulo, L. Silva, M. Seifrtová, C. Lino and P. Solich, Anal. Bioanal. Chem., 2010, 396, 2929-2936.

13 M. H. Khan, H. Bae and J. Y. Jung, J. Hazard. Mater., 2010, 181, 659-665.

14 Y. Wang, H. Zhang, J. H. Zhang, L. Chen, Q. Q. Huang, J. Wu and F. Liu, J. Hazard. Mater., 2011, 192, 35-43.

15 R. Acosta, V. Fierro, D. Y. A. Martinez, D. Nabarlatz and A. Celzard, Chemosphere, 2016, 149, 168-176.

16 T. T. Lu, X. X. Xu, X. X. Liu and T. Sun, Chem. Eng. J., 2017, 30, 8151-8159.

17 P. Nolwenn, O. Juan and A. Abdeltif, Bioresour. Technol., 2009, 100, 3769-3774.

18 K. L. Yang, Q. Y. Yue, J. J. Kong, P. Zhao, Y. Gao, K. F. Fu and B. Y. Gao, Chem. Eng. J., 2016, 285, 319-330.

19 B. E. Logan and J. M. Regan, Trends Microbiol., 2006, 14, 512518.
20 D. R. Lovley and K. P. Nevin, Curr. Opin. Biotechnol., 2011, 22, 441-448.

21 L. Wang, Y. L. Liu, J. Ma and F. Zhao, Water Res., 2016, 88, 322-328.

22 H. Liu, S. Cheng and B. E. Logan, Environ. Sci. Technol., 2005, 39, 658-662.

23 Y. J. Feng, X. Wang, B. E. Logan and H. Lee, Appl. Microbiol. Biotechnol., 2008, 78, 873-880.

24 T. S. Song, X. Y. Wu and C. C. Zhou, Bioprocess Biosyst. Eng., 2014, 37, 133-138.

25 Y. Luo, G. L. Liu, R. D. Zhang and C. P. Zhang, J. Power Sources, 2010, 195, 190-194.

26 S. Zhang, H. L. Song, X. L. Yang, K. Y. Yang and X. Y. Wang, Chemosphere, 2016, 164, 113-119.

27 S. Zhang, H. L. Song, X. L. Yang, K. Y. Yang and X. Y. Wang, RSC Adv., 2016, 6, 95999-96005.

28 M. Pérez-Moya, M. Graells, G. Castells, J. Amigó, E. Ortega, G. Buhigas, L. M. Pérez and H. D. Mansilla, Water Res., 2010, 44, 2533-2540.

29 L. Truong, S. L. Harper and R. L. Tanguay, Methods Mol. Biol., 2011, 691, 271-279.

30 H. L. Jiang, J. H. Tay, A. M. Maszenan and S. T. L. Tay, Appl. Environ. Microbiol., 2004, 70, 6767-6775.

31 W. K. Deng, Y. B. Wang, Z. X. Liu, H. Cheng and Y. Xue, PLoS One, 2014, 9, 111988.

32 A. F. Schier, Nature, 2013, 496, 443-444.

33 A. J. Peterson and R. T. Curr, Curr. Opin. Chem. Biol., 2014, 24, 58-70.

34 P. McGrath and C. Q. Li, Drug Discovery Today, 2008, 13, 394401.

35 D. R. Nielsen, P. J. McLellan and A. J. Daugulis, Biotechnol. Lett., 2006, 16, 1293-1298.

36 M. Ahmed and D. D. Focht, Can. J. Microbiol., 1973, 19, 4752.

37 L. Gumaelius, G. Magnusson, B. Pettersson and G. Dalhammar, Int. J. Syst. Evol. Microbiol., 2001, 51, 9991006.

38 K. Rabaey, N. Boon, S. D. Siciliano, M. Verhaege and W. Verstraete, Appl. Environ. Microbiol., 2004, 70, 5373-5382.

39 D. R. Bond and D. R. Lovley, Appl. Environ. Microbiol., 2003, 691, 548-1555.

40 R. P. Ryan, S. Monchy, M. Cardinale, S. Taghavi, L. Crossman, M. B. Avison, G. Berg, D. V. D. Lelie and J. M. Dow, Nat. Rev. Microbiol., 2009, 7, 514-525.

41 E. S. Anderson, Br. Med. J., 1965, 27, 1289-1291.

42 S. Kumar, V. R. Tripathi and S. K. Garg, J. Environ. Sci. Technol., 2013, 10, 789-798. 